\title{
3D Imaging of the Fermi Surface by Thermal Diffuse Scattering
}

\author{
A. Bosak, ${ }^{1}$ M. Hoesch, ${ }^{1}$ M. Krisch, ${ }^{1}$ D. Chernyshov, ${ }^{2}$ P. Pattison, ${ }^{2,3}$ C. Schulze-Briese, ${ }^{4}$ B. Winkler, ${ }^{5}$ V. Milman, ${ }^{6}$ \\ K. Refson, ${ }^{7}$ D. Antonangeli, ${ }^{8}$ and D. Farber ${ }^{9,10}$ \\ ${ }^{1}$ European Synchrotron Radiation Facility, BP 220, 38043 Grenoble Cedex, France \\ ${ }^{2}$ Swiss-Norwegian Beam Lines, BP 220, 38043 Grenoble Cedex, France \\ ${ }^{3}$ Laboratoire de Cristallographie, Ecole Polytechnique Fédérale de Lausanne, CH-1015 Lausanne, Switzerland \\ ${ }^{4}$ Paul Scherrer Institut, Swiss Light Source, 5232 Villigen-PSI Switzerland \\ ${ }^{5}$ Geowissenschaften, Goethe-Universität, Altenhoeferallee 1, D-60438 Frankfurt a.M., Germany \\ ${ }^{6}$ Accelrys, 334 Cambridge Science Park, Cambridge CB4 OWN, United Kingdom \\ ${ }^{7}$ Rutherford-Appleton Laboratory, Building R3, Chilton, Didcot, Oxfordshire OX11 0QX, United Kingdom \\ ${ }^{8}$ Institut de Minéralogie et de Physique des Milieux Condensés, UMR CNRS 7590, Institut de Physique du Globe de Paris, \\ Université Paris 6 et 7, 75005 Paris, France \\ ${ }^{9}$ Lawrence Livermore National Laboratory, Livermore, California 94550, USA \\ ${ }^{10}$ University of California, Santa Cruz, Santa Cruz, California 95064, USA
}

(Received 13 March 2009; published 12 August 2009)

\begin{abstract}
We use thermal diffuse scattering of $\mathrm{x}$ rays to visualize the lens-shaped portions of the Fermi surface in metallic zinc. Our interpretation of the nature of the observed scattered intensity anomalies is supported by the incorporation of inelastic x-ray scattering measurements as well as ab initio calculations of the electronic structure and lattice dynamics. Our work demonstrates that thermal diffuse scattering complements well-established techniques and is a powerful tool in its own right for studying the shape of the Fermi surface through the associated electron-phonon coupling.
\end{abstract}

DOI: 10.1103/PhysRevLett.103.076403

The Fermi surface-the surface in electron momentum space of a metal that separates occupied and empty electronic states-is one of the most important theoretical concepts in solid-state physics. Indeed, the shape of the Fermi surface controls the electric, magnetic, and thermal properties of materials. The most commonly used experimental techniques for the determination of the Fermi surface (FS) are the oscillation of transport properties in a magnetic field (i.e., the de Haas-van Alphen effect [1] and the Shubnikov-de Haas effect [2]) and angle resolved photoemission spectroscopy [3]. Two-photon positron annihilation [4] and Compton scattering [5] comprise more indirect methods of probing the topology of the Fermi surface. However, all the above-mentioned techniques present inherent limitations either in information content or in the potential applications. Interestingly, one of the earliest techniques proposed to study the Fermi surface was to search for abrupt variations in the dispersion of phonons [6]. These so-called Kohn anomalies originate from the singularity at $k=2 k_{F}$ in the static dielectric function of the conduction electrons, and therefore the Fourier transform of the pair-interaction potential, reflecting changes in the charge screening by the conduction electrons when the phonon wave vector $q$ exceeds the spanning across the Fermi surface $k=2 k_{F}$.

While numerous inelastic neutron scattering (INS), and later inelastic x-ray scattering (IXS) studies, documented these Kohn anomalies, only a few studies were devoted to actually mapping these Kohn anomalies in two or three dimensions [7-10]. As the phonon frequencies are typically determined point-by-point and branch-by-branch
PACS numbers: 71.18.+y, 63.20.-e, 64.70.kd

along the high-symmetry directions, the reconstruction of the full Fermi surface in three dimensions would be extremely time consuming with this approach. On the other hand, for some time it has been recognized that the intensity distribution of $\mathrm{x}$-ray scattering by thermally populated phonons (thermal diffuse scattering; TDS) also carries information on the phonon dispersions [11]. One of the downsides of this approach has been that the quantitative treatment of TDS data is much less straightforward than for INS and IXS, and is typically model dependent. However, the dispersion anomalies can be identified in a modelindependent fashion via characteristic intensity variations of the TDS. Nevertheless, there has been little work in this area [12-14], most of which is limited to 1D anomalies, and to a few high-symmetry points or high-symmetry planes. The advent of third generation synchrotron sources, in combination with efficient large area detectors, has led to a revival of TDS studies [15-18], as well as static diffuse scattering experiments, aimed at elucidating the local ordering and Fermi surface phenomena in disordered alloys $[19,20]$.

In the present work, we use synchrotron x-ray diffraction in conjunction with area detectors to visualize threedimensional lens-shaped diffuse objects in the Fermi surface of metallic zinc. By complementing our measurements with an inelastic x-ray scattering study and $a b$ initio calculations, we demonstrate that the observed diffuse scattering is of phonon-related nature and that the lens-shaped objects mimic the shape of the Fermi surface via electron-phonon interactions. We chose metallic zinc as a benchmark since the phonon dispersions along high- 
symmetry directions are well known from INS [9,21,22], and Kohn anomalies are easily observable in this material.

The TDS studies were performed in two runs, the first on the Swiss-Norwegian beam lines (SNBL) at the ESRF, and the second on beam line X06a at the Swiss Light Source. $\mathrm{X}$ rays were monochromatized to $\lambda=0.722 \AA$ and $\lambda=$ $0.710 \AA$, respectively, within a bandwidth of $\Delta \lambda / \lambda \sim 2 \times$ $10^{-4}$. A high quality, needlelike $50-\mu \mathrm{m}$-thick zinc single crystal (MaTecK $\mathrm{GmbH}$ ) was mounted on a rotation stage, and diffuse scattering patterns were recorded with an increment of $0.1^{\circ}$ over an angular range of $360^{\circ}$. Orientation matrix refinement and preliminary reciprocal space reconstructions were performed using the CRYSALIS software package (Oxford Diffraction, Ltd.). For the final reciprocal space reconstructions we applied corrections for polarization and for solid angle conversion associated with the planar projection. For 3D visualization purposes the reconstructed volume is averaged with its symmetrically equivalent orientations in respect of the Laue symmetry of the sample, thus improving the signal-to-noise ratio. Constant-intensity surfaces were traced from the generated 3D data array using the UCSF Chimera package and for the presentation purposes rendered in POV-Ray software [23]. The use of an energy discriminating pixel detector (PILATUS 6M [24]) was found undispensable to suppress the strong zinc fluorescence contribution.

The TDS results were complemented by IXS data, recorded on beam line ID28 at the ESRF, operating at a wavelength $\lambda=0.6968 \AA$ with an instrumental energy resolution of $3 \mathrm{meV}$ full width at half maximum (FWHM). Measurements were performed at room temperature in transmission geometry. The direction and size of the momentum transfer were selected by an appropriate choice of the scattering angle and the sample orientation in the horizontal scattering plane. The momentum resolution was set to $0.28 \mathrm{~nm}^{-1}$ and $0.84 \mathrm{~nm}^{-1}$ in the horizontal and vertical planes, respectively. Further details of the experimental setup and data treatment procedure can be found elsewhere [25].

$A b$ initio calculations of the Fermi surface (FS) of hep zinc were performed in the generalized-gradient approximation of density functional theory (GGA/DFT) using the all-electron WIEN2K code [26]. The linearized augmented plane-wave basis with local orbitals was expanded to $k_{\max }$ given by $r k_{\max }=7$ outside the atomic spheres with radius $r=2.38$ a.u. A symmetry reduced grid of $35 \times 35 \times 16$ points in momentum space was used for convergence of the total energy. An optimization of the unit cell dimensions gave $c / a=1.886$ and $a=2.68 \AA$, in good agreement with literature values $c / a=1.8561(2)$ and $a=$ 2.6644(3) [27]. The Fermi surface in the $\Gamma-K-H-A$ plane was extrapolated from Fermi level crossings of all bands in a $50 \times 30$ grid in the symmetry reduced part of the first Brillouin zone.

$A b$ initio lattice dynamics calculations were performed using DFT with a plane-wave basis set and pseudopoten- tials as implemented in the CASTEP code [28]. The PerdewBurke-Ernzerhof variant of the generalized-gradient approximation [29] was used for exchange and correlation, and pseudopotententials were of the Vanderbilt ultrasoft construction [30] with a plane-wave cutoff of $350 \mathrm{eV}$. Brillouin-Zone sampling used the method of Monkhorst and Pack [31] with a distance of less than $0.009 \AA^{-1}$ between grid points. The finite-difference supercell method [32] was applied; a $4 \times 4 \times 3$ supercell based on the experimental lattice parameters was used, and the force constant matrix was transformed to construct dynamical matrices using the formula of Gonze and Lee [33] and diagonalized throughout the Brillouin zone to yield phonon frequencies and eigenvectors. Consequent TDS calculations followed well-established formalism [15] with an isotropic Debye-Waller factor [34].

Figure 1(a) shows the reconstructed experimentally derived diffuse scattering intensity in the $H H L$ and $H O L$ planes. Besides the thermal diffuse scattering from longwavelength acoustic phonons, dominating in the close vicinity of the Bragg reflections, additional well-defined features are clearly visible. sixfold (HK2 layer) or (approximately) threefold ( $H K 3$ layer) intensity variations

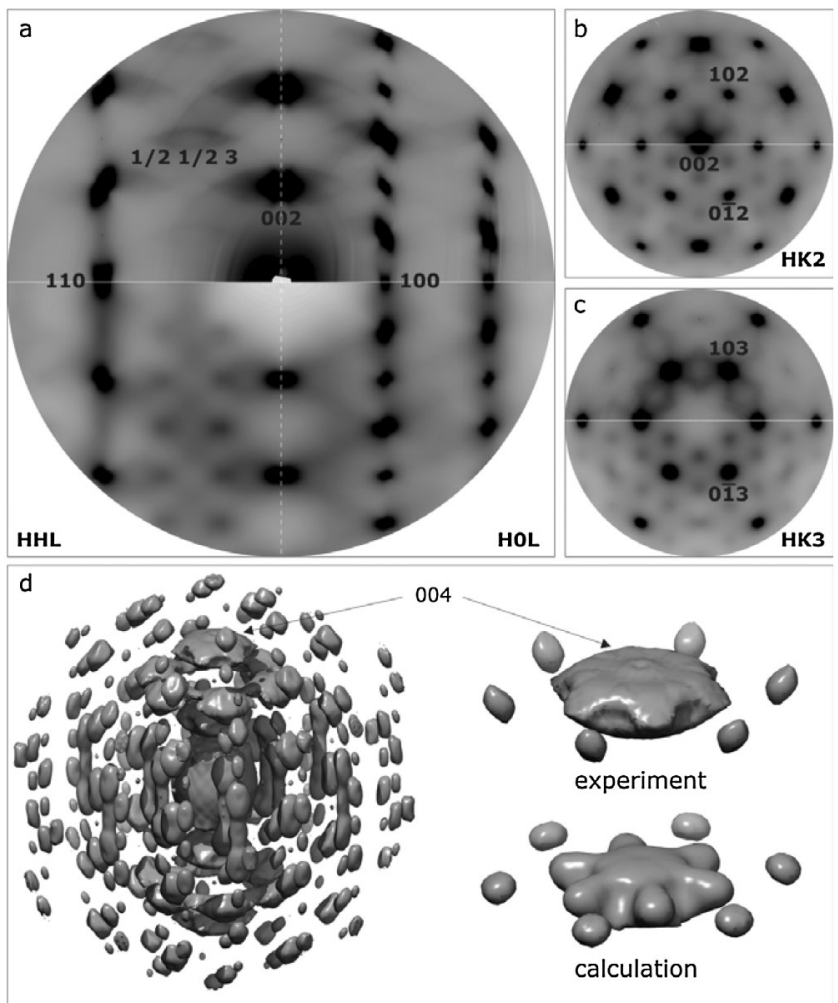

FIG. 1. Reciprocal space maps for zinc. Sections along the $c^{*}$ axis: (a) $H H L$ and $H 0 L$, and perpendicular to it: (b) $H K 2$ and (c) $H K 3$. The upper part of panels show the experimental pattern, and the lower part the calculated pattern. (d) Constantintensity surfaces for diffuse scattering: general view of the experimental data set and comparison of experiment and theory for the region around the (004) Bragg spot. For visualization purposes $a \cos ^{2}(2 \theta)$ factor is added. 
[Figs. 1(b) and 1(c), respectively] around Bragg spots reflect both the crystallographic structure and the anisotropic dispersion of acoustic phonons [22]. The expected monotonically varying TDS intensity is additionally modulated, clearly showing the lenslike objects with an axial symmetry centered on intense Bragg spots. Their boundaries are characterized by sharp intensity variation and are easy to locate. We furthermore observe hollow lenslike objects of smaller size [i.e., centered around $\left.\left(\begin{array}{lll}1 & \frac{1}{2} & 3\end{array}\right)\right]$. As no structural disorder is expected for a chemically pure zinc single crystal, we relate the observed lenslike diffuse objects to portions of the Fermi surface. These are visualized via nonmonotonic intensity variations in the thermal diffuse scattering, and correspond to the occurrence of a Kohn anomaly in the phonon dispersion. The contrast is particularly favorable when the phonon wave vector translates, or nests, parallel pieces of the Fermi surface. We obtain a value of $q \approx 3.14 \AA^{-1}$ for the radius of curvature of the large lenslike objects [i.e., centered around $\left(\begin{array}{lll}0 & 0 & 2 n\end{array}\right)$ spots], yielding a value of $q / 2 \approx k_{F} \approx 1.57 \AA^{-1}$, in close agreement with the corresponding value for a free electron model $\left(1.573 \AA^{-1}\right.$ if two conduction electrons are attributed to each zinc atom). A 3D representation of constantintensity surfaces is shown in Fig. 1(d), where lenslike objects with sixfold modulation can be easily identified along the $c^{*}$ axis.

Our experimental observations are further supported by $a b$ initio calculations of the Fermi surface (Fig. 2). As Fig. 2(b) reveals, the large lenslike objects correspond to the inner part of the Fermi surface around the $\Gamma$ point. The shape of this part of the Fermi surface mimics the corresponding surface of dispersion anomaly locations (Kohn surface [35] - in the case of zinc it has a well-defined geometrical meaning) through its homothetic transforma-
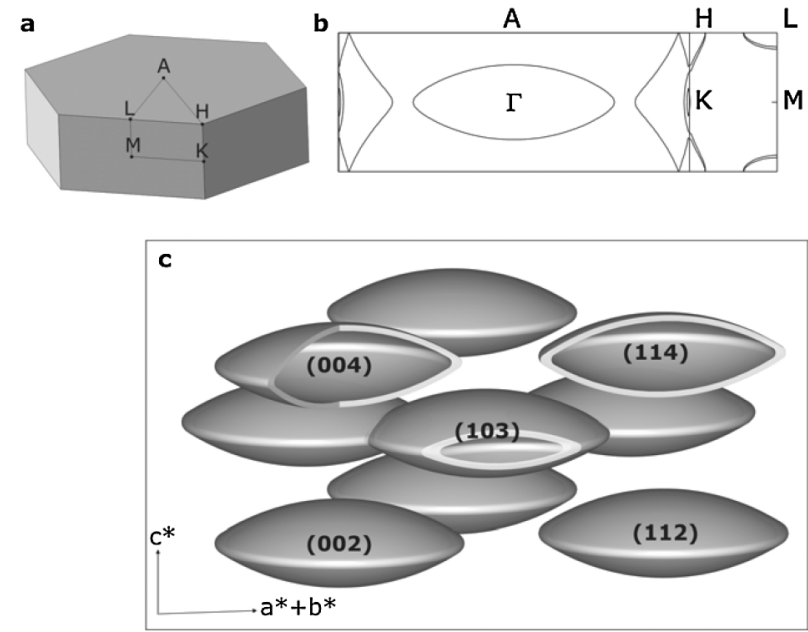

FIG. 2. (a) Brillouin zone of zinc with denoted special points; (b) high-symmetry section of the Fermi surface obtained by $a b$ initio calculations; (c) scheme of the assembly of lenslike surfaces centered on strong Bragg spots and their dissection by HHL plane. tion with the scaling factor $2\left(q=2 k_{F}\right)$. This gives us the opportunity to visualize the shape of the Fermi surface in a straightforward way. The hollow lenslike objects of smaller size, observed in $H H L$ sections [i.e., centered around $\left.\left(\frac{1}{2} \frac{1}{2} 3\right)\right]$ are in fact sections of the large "lenses" [Fig. 2(c)].

Further evidence in favor of the phonon-mediated nature of the observed anomalies is provided by the complementary IXS study and theoretical lattice dynamics calculations. The IXS experiment revealed both phonon softening - in accordance with the INS data [22]and pronounced intensity changes across the Kohn surface (Fig. 3). The calculation of diffuse scattering maps from CASTEP-generated eigenvalues and eigenvectors of the dynamical matrix could be performed using the well-established formalism [15]. The lower parts of Figs. 1(a)-1(d) present the corresponding maps and constant-intensity surfaces. While the exact shape and sharpness of Kohn anomalies are not reproduced, the overall resemblance in terms of contrast, symmetry, and intensity distribution is satisfactory. It is intriguing to note that the experimentally observed features (recorded at room temperature) associated with the Kohn surface appear sharper than in our zero Kelvin athermal calculations. This could be due to the violation of the adiabatic BornOppenheimer approximation (ABO), which states that the lighter electrons adjust adiabatically to the motion of the heavier nuclei, therefore remaining at any time in their ground state. The ABO is well justified when the energy gap between ground and excited electronic states is larger than the energy scale of the nuclear motion, which is obviously not the case for strong electron-phonon coupling [36].

Our proof-of-principle demonstration underlines the great potential of TDS in the study of electron-phonon coupling and Fermi surface phenomena. The 3D shape of

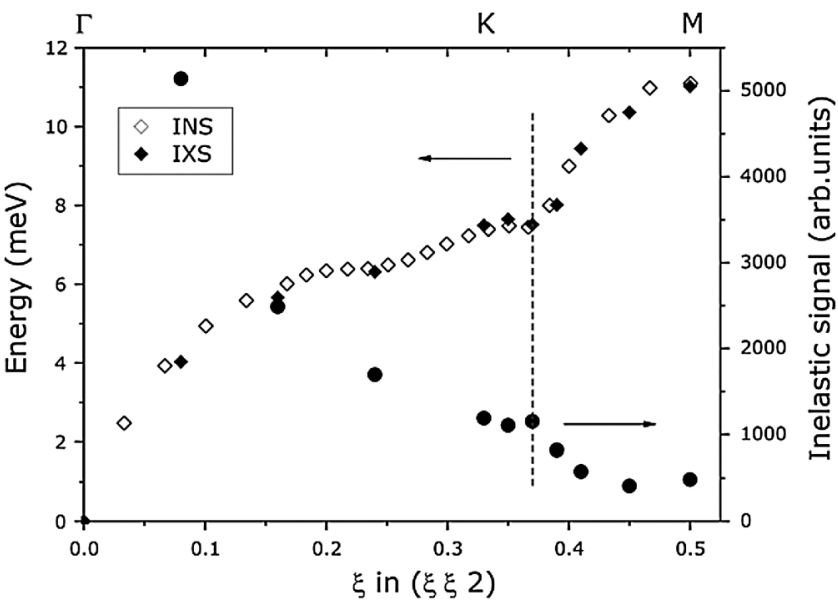

FIG. 3. Dispersion of transverse acoustic phonons of zinc propagating along $\Gamma-K-M$ direction and polarized along $\Gamma-A$ direction: comparison of IXS and INS [22] data (left axis); corresponding evolution of the inelastic signal intensity scattered on TA phonon (right axis). 
the Kohn surface can be reconstructed within the time scale of hours using synchrotron $\mathrm{x}$-ray diffraction equipment. The full potential of TDS can only be efficiently exploited if complementary atomistic model calculations are available. In the present study, we have used such calculations to remove ambiguities in the data interpretation, namely, to demonstrate that the observed features are entirely caused by phonons, but more advanced treatments would allow to also extract information about the strength of electronphonon coupling, i.e., employing density functional perturbation theory. The main limitation of the approach presented here is the requirement that scattering by the sample is not dominated by quasielastic scattering due to dynamic processes or disorder. If this condition is satisfied, diffuse scattering maps can be probed together with Bragg intensities in a single experiment, thus providing both static structure and phonon dynamics. It is important to emphasize that by using an insertion device synchrotron beam line and energy-selective area detectors, the data quality and data collection efficiency are increased by orders of magnitude comparing to standard equipment. Accordingly, TDS experiments provide a powerful experimental approach for Fermi surface studies that can be exploited in a wide range of experimental settings where the traditional techniques cannot due to experimental constraints. For example, the de Haas-van Alphen and Shubnikov-de Haas effects cannot be used if the sample exhibits a lowtemperature insulating phase, or if the electronic structure is altered by the applied magnetic field. The use of angleresolved photoemission spectroscopy is typically restricted to the study of surfaces as the escape depth of electrons does not exceed the topmost atomic layers in the commonly employed photon energy range. The electronic properties of surfaces may differ significantly from those of the bulk due to surface reconstruction, or due to differences in the stoichiometry of the bulk and surface, which may be induced during sample preparation or due to the required ultrahigh vacuum environment. On the contrary, TDS experiments can have wide application for high pressure studies in diamond anvil cells where one can use the fine $\mathrm{x}$-ray focus to probe small single crystals or single crystal domains within a polycrystalline sample. The TDS approach we lay out here lends itself as well to time resolved studies, which are also outside the range of possible investigation with the classical experimental approaches.

[1] W. J. de Haas and P. M. van Alphen, Proc. R. Netherlands Acad. Arts Sci. 33, 1106 (1930).

[2] L. W. Schubnikow and W. J. de Haas, Proc. R. Netherlands Acad. Arts Sci. 33, 130 (1930).

[3] S. Hüfner, in Photoelectron Spectroscopy (SpringerVerlag, Berlin, 1995).

[4] L. Oberli, A. A. Manuel, R. Sachot, P. Descouts, and M. Peter, Phys. Rev. B 31, 6104 (1985).
[5] S. B. Dugdale et al., Phys. Rev. Lett. 96, 046406 (2006).

[6] W. Kohn, Phys. Rev. Lett. 2, 393 (1959).

[7] R. I. Sharp, J. Phys. C 2, 432 (1969).

[8] R. Stedman and G. Nilsson, Phys. Rev. Lett. 15, 634(1965).

[9] J. W. Weymouth and R. Stedman, Phys. Rev. B 2, 4743 (1970).

[10] P. K. Iyengar, G. Venkataraman, Y. H. Gameel, and K. R. Rao, in Proceedings of the Symposium on Neutron Inelastic Scattering, Copenhagen (IAEA, Vienna, 1968), Vol. I, pp. 195-201.

[11] K. Lonsdale, Crystals and X-Rays (G. Bell, London, 1948).

[12] A. Paskin and R. J. Weiss, Phys. Rev. Lett. 9, 199 (1962).

[13] J. M. Costello and J. W. Weymouth, Phys. Rev. 184, 694 (1969).

[14] Y. Yamada, J. C. Tsang, and G. V. Subba-Rao, Phys. Rev. Lett. 34, 1389 (1975).

[15] R. Xu and T.-C. Chiang, Z. Kristallogr. 220, 1009 (2005).

[16] M. Holt, M. Sutton, P. Zschack, H. Hong, and T.-C. Chiang, Phys. Rev. Lett. 98, 065501 (2007).

[17] M. Holt, P. Zschack, H. Hong, M. Y. Chou, and T.-C. Chiang, Phys. Rev. Lett. 86, 3799 (2001).

[18] J. Wong et al., Appl. Phys. Lett. 84, 3747 (2004).

[19] S. C. Moss, Phys. Rev. Lett. 22, 1108 (1969).

[20] H. Reichert, O. Klein, V. Bugaev, O. Shchyglo, A. Udyanskyy, H. Dosch, and K. F. Peters, Phys. Rev. Lett. 90, 185504 (2003).

[21] E. Maliszewski, J. Rosolowski, D. Sledziewska, and A. Czachor, in Proceedings of the Conference on Lattice Dynamics, edited by R.F. Wallis (Pergamon Press, Oxford, 1965), pp. 33-37.

[22] L. Almqvist and R. Stedman, J. Phys. F 1, 785 (1971).

[23] http://www.povray.org

[24] C. Brönnimann et al., J. Synchrotron Radiat. 13, 120 (2006).

[25] M. Krisch and F. Sette, in Light Scattering in Solids, Novel Materials and Techniques, Topics in Applied Physics 108 (Springer-Verlag, Berlin, Heidelberg, 2007).

[26] P. Blaha, K. Schwarz, G. K. H. Madsen, D. Kvasnicka, and J. Luitz, WIEN2K, An Augmented Plane Wave + Local Orbitals Program for Calculating Crystal Properties (Karlheinz Schwarz, Techn. Universität Wien, Austria, 2001).

[27] J. Donohue, The Structures of the Elements (Wiley, New York, 1974), pp. 225-230.

[28] S. J. Clark et al., Z. Kristallogr. 220, 567 (2005).

[29] J. P. Perdew, K. Burke, and M. Ernzerhof, Phys. Rev. Lett. 77, 3865 (1996).

[30] D. Vanderbilt, Phys. Rev. B 41, 7892 (1990).

[31] H. J. Monkhorst and J. D. Pack, Phys. Rev. B 13, 5188 (1976).

[32] K. Kunc and R. M. Martin, Phys. Rev. Lett. 48, 406 (1982).

[33] X. Gonze and C. Lee, Phys. Rev. B 55, 10355 (1997).

[34] H. X. Gao and L.-M. Peng, Acta Crystallogr. Sect. A 55, 926 (1999).

[35] J. W. Weymouth and R. Stedman, Phys. Rev. B 2, 4743 (1970).

[36] S. Pisana, M. Lazzeri, C. Casiraghi, K. S. Novoselov, A. K. Geim, A. C. Ferrari, and F. Mauri, Nature Mater. 6, 198 (2007). 